\title{
Exercício físico e síndrome metabólica
}

\author{
Physical exercise and metabolic syndrome
}

\author{
Fabrício V. A. Vasconcellos \\ Luiz G. Kraemer-Aguiar \\ Ada Fernanda P. S. Lima
}

Tânia M. P. F. Paschoalino

Walace D. Monteiro*

\section{Resumo}

A síndrome metabólica (SM) é caracterizada por um conjunto de fatores de risco para o desenvolvimento de doenças cardiovasculares e de diabetes mellitus tipo 2 (DM2), que se manifestam, em geral, mais concomitantemente do que isoladamente, e estão relacionados à obesidade (particularmente central), a elevações da pressão arterial, da glicemia e dos triglicerídeos e aos baixos níveis de HDLcolesterol. Nas últimas décadas, o número de pessoas acometidas por um ou mais dos fatores de risco para SM tem aumentado drasticamente, tornando essa síndrome um problema de saúde pública em escala mundial. O tratamento da SM pode ser feito através de diferentes terapias, envolvendo recursos medicamentosos e não medicamentosos. No caso da terapia não medicamentosa, o exercício físico tem sido apontado como um importante instrumento, por atuar direta ou indiretamente no combate a todos os fatores de risco que compõem a SM. Desta forma, a presente revisão aborda o papel

Revista HUPE, Rio de Janeiro, 2013;12(4):78-88 doi:10.12957/rhupe.2013.8715 da prática sistemática do exercício nos diferentes fatores de risco para SM. Inicialmente, são enfocados os efeitos do exercício na obesidade, destacando-se nesse contexto a obesidade central. Em seguida, são apresentados os efeitos do exercício sobre o LDL-, HDL-colesterol e triglicerídeos. Posteriormente, são tecidas considerações sobre a aplicação do exercício na hipertensão arterial, resistência à insulina e DM2. Por fim, o texto descreve os aspectos metodológicos que devem reger a prescrição de exercício aeróbio, de força muscular e de flexibilidade para indivíduos portadores de SM.

Descritores: Síndrome metabólica; Exercício físico; Obesidade; Dislipidemias; Hipertensão; Diabetes mellitus tipo 2.

\footnotetext{
Abstract

The metabolic syndrome (MS) is characterized by a cluster of risk factors for cardiovascular diseases and type 2 diabetes mellitus (T2DM) that occur together more often than alone by

"Endereço para correspondência: Laboratório de Atividade Física e Promoção da Saúde, UERJ. Rua São Francisco Xavier, 524, sala 8.121, bloco F Rio de Janeiro, RJ, Brasil. CEP: 20550-013. Telefone: 5521 2334-0775 E-mail: walacemonteiro@uol.com.br
} 
chance, commonly associated with obesity (particularly central obesity), elevated blood pressure and triglycerides, low levels of HDLcholesterol and disglycemia. In the last decades, the number of individuals affected by more than one of the risks factors for MS has increased severely, turning this syndrome into a public health problem worldwide. The treatment for MS should be performed by different therapies that involve medical resources and lifestyle interventions. In respect to lifestyle changes, physical exercise has been indicated as an important alternative that acts directly or indirectly on all risk factors comprising the MS. Thus, the present review explores the role of systematic physical exercise practice on different risk factors for MS. Initially we focus on the effect of physical exercise on obesity, especially on the abdominal one. Subsequently the effects of physical exercise on LDL- and HDL-cholesterols and triglycerides are shown. Additionally, we presented some considerations on the benefits of exercise on blood pressure, insulin resistance and T2DM. Finally, we stated the methodological guidelines for prescription of aerobic exercise, muscular strength and flexibility for individuals with MS.

Keywords: Metabolic syndrome; Exercise; Obesity; Dyslipidemias; Hypertension; Diabetes mellitus, type 2.

\section{Introdução}

A prevalência de síndrome metabólica (SM) tem crescido rapidamente, tanto em países desenvolvidos como em países em desenvolvimento, e normalmente está associada à da obesidade. ${ }^{1}$ Estudos epidemiológicos em adultos indicam que nos Estados Unidos a prevalência da SM estaria entre $20,5 \%$ e $26,7 \%$, na Europa entre $19,8 \%$ e $24 \%$, enquanto no Brasil entre $18 \%$ e $30 \%$, apresentando ainda uma correlação positiva com a idade. ${ }^{2}$ A SM pode ser descrita como a associação de diversos fatores de riscos para doenças cardiovasculares (DCV) e DM2, como: obesidade central, hipertrigliceridemia, disglicemia, dislipidemia e hipertensão arterial. Além disso, devemos ressaltar que as DCVs representam a maior causa de mortalidade no mundo moderno. ${ }^{3}$ Os critérios para diagnóstico da SM foram estabelecidos por diferentes organizações e sofrem algumas variações, o que acaba por influenciar as diferentes prevalências da síndrome em todo o mundo. Em 1998, os critérios para caracterizar a SM foram definidos pela Organização Mundial da Saúde (OMS) (Quadro 1), incluindo, além da hipertensão arterial e da dislipidemia, também a obesidade e a microalbuminúria. ${ }^{4}$

Em 2001, o National Institute of Health (NIH), por meio do National Cholesterol Education Program (NCEP), reuniu o Third Adult Treatment Panel (ATP III) ${ }^{5}$ e sugeriu um novo critério para caracterizar a SM (Quadro 2). Neste, nenhum dos componentes da síndrome apresentava superioridade sobre qualquer outro e o diagnóstico é feito quando três ou mais dos fatores de risco estiverem presentes. Posteriormente, em 2005, a International Diabetes Federation (IDF) recomendou uma nova definição (Quadro 3), na qual a obesidade central, avaliada pela medida da circunferência abdominal (CA), passou a ter maior importância para o diagnóstico. ${ }^{6}$ Com isso, para o diagnóstico da SM, a presença de uma CA elevada é essencial e esta deve estar associada a dois ou mais fatores de risco (Quadro 2). Ademais, a medida da CA passou a ser um método de triagem simples e de fácil aplicabilidade clínica. O grupo latino-americano não foi investigado e recomendaram o emprego dos mesmos pontos de corte da CA empregados nos asiáticos. A falta de uniformidade nestes pontos de corte foi um dos motivos que congregou diversos especialistas em 2009. ${ }^{7}$ Estes, representados por diversas associações, publicaram o Joint Interim Statement, um consenso para o diagnóstico da SM, no qual sugeriram que para cada grupo étnico deveríamos empregar pontos de corte específicos para a CA e previamente propostos pela IDF. Entretanto, para os outros 
Quadro 1. Critérios diagnósticos para síndrome metabólica propostos pela Organização Mundial de Saúde (OMS, 1998)

\begin{tabular}{|c|c|}
\hline \multicolumn{2}{|c|}{ Presença de 3 ou mais dos seguintes critérios: } \\
\hline Obesidade & Relação cintura/quadril $>0,9$ em homens e $>0,85$ em mulheres e/ou IMC $>30 \mathrm{~kg} / \mathrm{m}^{2}$ \\
\hline Glicose ( $\geq 110 \mathrm{mg} / \mathrm{dl}$ ) & Diabetes, intolerância à glicose ou resistência insulínica comprovada pelo clamp* \\
\hline Triglicerídeos & $\geq 150 \mathrm{mg} / \mathrm{dl}$ \\
\hline HDL & $<35 \mathrm{mg} / \mathrm{dl}$ em homens e $<39 \mathrm{mg} / \mathrm{dl}$ em mulheres \\
\hline Pressão arterial & Pressão sistólica $\geq 140 \mathrm{mmHg}$ ou diastólica $\geq 90 \mathrm{mmHg}$, ou tratamento para HA \\
\hline Outros & Excreção urinária de albumina $\geq 20 \mathrm{mcg}$ ou relação albumina/creatinina $\geq 30 \mathrm{mg} / \mathrm{g}$ \\
\hline
\end{tabular}

*Dois fatores e obrigatoriamente o componente assinalado

Quadro 2. Critérios diagnósticos para síndrome metabólica propostos pelo Third Adult Treatment Panel (ATP III, 2001)

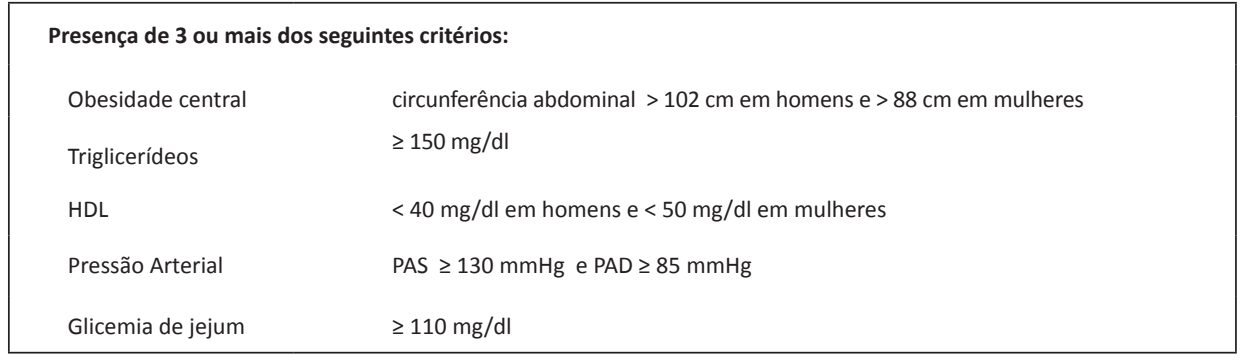

Quadro 3. Critérios diagnósticos para síndrome metabólica, propostos pela International Diabetes Federation (IDF, 2005)

\begin{tabular}{|c|c|c|}
\hline \multicolumn{3}{|c|}{ Circunferência abdominal, de acordo com sexo e etnia: } \\
\hline & Homens $(\mathrm{cm})$ & Mulheres $(\mathrm{cm})$ \\
\hline Norte-americanos & 102 & 88 \\
\hline Europeus & 94 & 80 \\
\hline Sul-asiáticos/chineses & 90 & 80 \\
\hline Sul-americanos/africanos & 90 & 80 \\
\hline Japoneses & $\underline{85}$ & 90 \\
\hline \multicolumn{3}{|c|}{ Associada à presença de 2 ou mais dos seguintes critérios: } \\
\hline Triglicerídeos & \multicolumn{2}{|c|}{$\geq 150 \mathrm{mg} / \mathrm{dl}$} \\
\hline HDL & $\leq 40 \mathrm{mg} / \mathrm{dl}$ & $\leq 50 \mathrm{mg} / \mathrm{dl}$ \\
\hline Pressão arterial & \multicolumn{2}{|c|}{ sistólica $\geq 135 \mathrm{mmHg}$ ou diastólica $\geq 85 \mathrm{mmHg}$} \\
\hline Glicemia de jejum & \multicolumn{2}{|c|}{$\geq 100 \mathrm{mg} / \mathrm{dl}$} \\
\hline
\end{tabular}

componentes da SM, os pontos de corte foram uniformizados independente do grupo étnico investigado (Quadro 3).

$\mathrm{O}$ acúmulo de tecido adiposo abdominal é uma das características mais marcantes da SM.
Este tecido produz e secreta peptídeos e proteínas envolvidos na inflamação e resposta do sistema imune e denominados adipocitocinas. $\mathrm{Na}$ obesidade abdominal, as concentrações de várias adipocitocinas encontram-se elevadas e têm sido 
relacionadas à hipertensão (angiotensinogênio), à inibição da fibrinólise (inibidor do ativador de plasminogênio-1), à resistência à insulina (fator de necrose tumoral- $\alpha$, interleucina-6 e resistina) e ao início ou progressão da lesão aterosclerótica (proteína C-reativa). ${ }^{8}$ De forma contrária, a adiponectina, um peptídeo com propriedades anti-inflamatórias e antiaterogênicas, correlaciona-se inversamente com o IMC e com o percentual de tecido adiposo. ${ }^{9}$ Desse modo, essas associações indicam haver uma relação causal entre obesidade e aterosclerose. Além disso, esses fatores relacionam-se não só em adultos, como também em crianças e adolescentes, na forma da alteração fisiopatológica mais precoce do processo aterosclerótico, denominada disfunção endotelial. ${ }^{10}$ Em concomitância com a elevação de adipocitocinas pró-aterogênicas, a SM tem em sua base fisiopatológica a ocorrência de resistência insulínica (RI). Esta está envolvida na etiopatogenia da hipertensão arterial, da elevação dos níveis de triglicérides e redução do HDL-colesterol e ainda das alterações do metabolismo da glicose presentes na SM. Além disso, a RI relaciona-se positivamente com a disfunção endotelial e é fator de risco independente para DCV.

Alimentação inadequada e inatividade física em indivíduos com predisposição genética são aspectos preponderantes para o surgimento da SM. Independentemente dos critérios adotados para diagnóstico da SM é consenso que se deve investigá-la, principalmente em indivíduos com excesso de peso, e instituir o tratamento de cada fator de risco identificado. Desta forma, para a prevenção dos fatores de risco ainda ausentes e a melhora daqueles já manifestos em portadores de SM, especial foco no combate à inatividade física tem um papel central, já que a SM, por ser considerada uma doença crônica não transmissível e de evolução silenciosa, a promoção da prática dos exercícios físicos tem efeitos benéficos comprovados.

Apesar da obesidade atualmente não compor um dos critérios para a SM, o emprego de uma dieta equilibrada e de atividades físicas regulares são duas das estratégias fundamentais no tratamento desta condição. Considerado como uma importante estratégia para aumentar o gasto energético, a atividade física regular está associada com a redução no IMC e no percentual de gordura, melhor aptidão cardiorrespiratória e de variáveis hemodinâmicas, redução na incidência dos componentes da SM e ainda em ganhos em aspectos psicológicos e socioafetivos. ${ }^{11} \mathrm{No}$ entanto, o que se pode observar atualmente é um incremento na prevalência de sedentarismo da população de maneira geral. Desta forma, a prática de exercícios ganha cada vez mais importância, no sentido de proporcionar ao praticante um estilo de vida saudável, diminuindo assim as chances de desenvolver a SM. Com base nestes preceitos, o presente estudo tem como objetivo revisar o efeito da atividade física sobre o excesso de peso, a dislipidemia, a hipertensão arterial e a resistência à insulina e disglicemia presentes na SM.

\section{Sobrepeso e obesidade}

Tem sido muito bem relatado na literatura a importância da atividade física no combate e prevenção do sobrepeso e obesidade e suas comorbidades. Estas evidências são razoavelmente consistentes, como pôde ser observado em diversas pesquisas. ${ }^{12}$ Demonstrouse inclusive que indivíduos com IMC elevado porém fisicamente ativos, apresentavam um estado de saúde melhor do que indivíduos com peso normal sedentários. ${ }^{12}$

A determinação do percentual de gordura através das medidas de dobras cutâneas é encontrada com mais frequência nos programas de intervenção em indivíduos obesos. Embora a maioria dos estudos tenha demonstrado uma redução do percentual de gordura, IMC e circunferência da cintura, a análise destas variáveis deve ser interpretada com algum cuidado. As diversas abordagens antropométricas, os diferentes métodos de avaliação e variados pontos de corte para 
a determinação da obesidade, dificultam a comparação entre os resultados dos diferentes estudos. Entretanto, pesquisas realizadas com delineamento randomizado e controlado ou com medidas sofisticadas, como a DEXA (dual-energy $X$-ray absorptiometry), também têm relatado uma diminuição significativa do percentual de gordura corporal, após a aplicação de um programa de exercício. ${ }^{13}$ Nesse sentido, cabe notar que um estudo recentemente realizado sugeriu que a ineficácia de algumas intervenções em indivíduos obesos estaria associada a uma falta de controle rígido nos critérios e métodos de avaliação da composição corporal. $^{14}$

Embora o excesso de peso seja um fator de risco para SM, nem todo indivíduo com sobrepeso ou obesidade tem um fenótipo considerado metabolicamente ativo e neste contexto não terá o diagnóstico de SM. Neste sentido, a obesidade tem sido mal definida como um fator de risco modificável em comparação com outros fatores, como hipertensão arterial, tabagismo e colesterol. ${ }^{15}$ Contudo, alguns estudos têm demonstrado que a obesidade intraabdominal ou visceral associa-se ao fenótipo metabolicamente ativo e a ocorrência de SM e está significativamente relacionada à DCV e ao diabetes mellitus tipo 2. Nesse padrão de obesidade, os níveis plasmáticos de proteína C-reativa (PCR) - marcador inflamatório considerado como fator preditivo de infarto do miocárdio - encontravam-se mais elevados do que em indivíduos que não apresentam a obesidade visceral. ${ }^{16}$

Alguns estudos também têm associado a obesidade visceral com disfunção endotelial e inflamação de baixo grau, assim como com risco aumentado de morbidades para doença coronariana e aterosclerótica. ${ }^{17}$ Entretanto, ainda existe alguma incongruência nesses resultados, tendo em vista que há evidências de que a atividade física seria capaz de reduzir marcadores inflamatórios, apesar de algumas pesquisas não terem confirmado tal efeito. ${ }^{18}$ Talvez estas divergências ocorram em função de variáveis potencialmente intervenientes, que devem ser controladas durante a elaboração de um programa de atividade física, por exemplo, tipo de exercício, frequência semanal, intensidade e duração do programa, entre outros fatores. No tocante à função endotelial, a maioria dos estudos relata efeito benéfico da atividade física. Desse modo, sugere-se que o exercício físico exerça um efeito direto e positivo na função vascular, provavelmente devido ao aumento na biodisponibilidade de óxido nítrico decorrente do estresse por cisalhamento (shear stress) durante o esforço. ${ }^{19}$

\section{Dislipidemia}

A dislipidemia da SM confere elevado risco cardiovascular e se caracteriza por aumento do nível sérico dos triglicerídeos, diminuição da fração HDL-colesterol e alterações qualitativas da fração LDL-colesterol com elevação dos níveis de partículas de LDL pequenas e densas com elevado potencial aterogênico. Este padrão dislipidêmico está associado particularmente à obesidade abdominal e à aterosclerose. ${ }^{19,10}$ Apesar do LDL-colesterol não compor um dos critérios para o diagnóstico da SM, estudos clínicos controlados apontam para a necessidade de sua redução como meta primária a ser alcançada, concomitantemente à correção dos níveis do HDL-colesterol e triglicerídeos. ${ }^{21}$

A literatura tem demonstrado que o exercício acarreta pouca ou nenhuma redução nos níveis de LDL-colesterol. ${ }^{21,22}$ Contudo, trabalhos que revisaram o assunto são consensuais em assumir a importância do exercício, por sua ação cardioprotetora, através de mudanças na composição química do LDL-colesterol demonstradas por aumento do colesterol livre, de ésteres de colesterol, de fosfolipídios e ainda da relação lipídios/proteína. ${ }^{18}$

No que diz respeito à fração HDL-colesterol, a prática do exercício tem se mostrado eficaz em promover seu aumento. ${ }^{22,23} \mathrm{O}$ HDL-colesterol possui a função de receptor final no transporte do colesterol dos tecidos para o fígado, remoção de lipídios oxidados do LDL-colesterol, inibição 
da fixação de moléculas de adesão (radicais livres) e de monócitos ao endotélio vascular e estimulação da liberação de óxido nítrico. ${ }^{24}$ Estudo realizado por Kelley e Kelley ${ }^{25}$ mostrou que a atividade física aeróbia aumenta em 11\% HDL-colesterol em adultos, o que enfatiza o papel benéfico da atividade física na SM e na redução do risco cardiovascular. Contudo, é importante destacar que aspectos genéticos afetam a magnitude das modificações nesta fração do colesterol. ${ }^{22}$ Quanto à concentração de triglicerídeos plasmáticos, esta encontra-se em geral diminuída após o treinamento físico. ${ }^{26} \mathrm{Em}$ revisão sobre o assunto, Nunes e colaboradores ${ }^{27}$ destacaram que a redução na concentração de triglicerídeos após o treinamento é observada em indivíduos previamente inativos e com triglicerídeos elevados no jejum, enquanto aqueles com baixos níveis de triglicerídeos exibiriam menor declínio.

A atividade física aeróbia regular constitui medida auxiliar no controle das dislipidemias, promovendo redução dos níveis plasmáticos de triglicerídeos e aumento dos níveis de HDL. Apesar de não apresentar alterações significativas para os níveis de LDL, pode ser um importante fator para o aumento no tamanho das moléculas de LDL, diminuindo sua capacidade de penetrar no espaço subendotelial vascular, de ser oxidado e consequentemente seu poder aterogênico. Contudo, para que os efeitos positivos do exercício possam ser adquiridos, o mesmo deve ser feito com regularidade, obedecendo a princípios metodológicos adequados. No caso do treinamento de força, este parece não ser efetivo em produzir alterações lipoproteicas favoráveis. Todavia, em nossa concepção, o aprimoramento da força e resistência muscular pode ser útil para melhorar a aptidão física geral, aumentando as possibilidades de se realizar o exercício aeróbio e atividades diárias, o que por si só representa um importante ganho para o indivíduo com SM.

\section{Hipertensão arterial}

A hipertensão arterial é caracterizada como uma doença silenciosa e de difícil intervenção precoce, pois sua instalação é, em sua maioria, assintomática. Além disso, algumas outras razões são apontadas para as baixas taxas de controle dessa doença, como o acesso precário a cuidados de saúde, aumento da prevalência de obesidade e falta de adesão, em longo prazo, às terapias. ${ }^{28}$

A prática regular de atividade física ou níveis moderados a elevados de aptidão física têm sido associados à redução da mortalidade cardiovascular, bem como à redução das pressões arteriais, sistólica e diastólica. Porém, os efeitos benéficos são dependentes da manutenção em um programa de exercícios. Nesse sentido, diversos estudos investigaram a relação entre prática da atividade física e melhora dos níveis pressóricos. Barroso e colaboradores ${ }^{29}$ avaliaram a influência da atividade física programada na pressão arterial de idosos sob tratamento não medicamentoso, concluindo que a atividade física supervisionada foi mais eficiente em manter o controle pressórico em comparação com grupo-controle sedentário. Assim, do mesmo modo que a atividade física pode apresentar efeitos benéficos no tocante à redução da resistência à insulina, esses benefícios são naturalmente estendidos à pressão arterial em indivíduos de todas as idades, principalmente porque na fisiopatologia da hipertensão na SM a resistência à insulina é fator causal. ${ }^{30}$

Em se tratando de crianças e adolescentes, dados evidenciam que a obesidade infantil tem crescido drasticamente nas últimas décadas. Sendo assim, a síndrome metabólica pediátrica também merece atenção. Rocchini e colaboradores ${ }^{31}$ investigaram crianças e adolescentes de 10-17 anos, submetidos a um programa multidisciplinar de perda de peso durante 20 semanas, com intervenção nutricional e 40 minutos de atividade aeróbia realizada três vezes por semana. O grupo de intervenção apresentou redução significativa nos valores de PAS e PAD em repouso, em comparação com o grupo-controle, mostrando que em crianças os resultados na redução da PA são semelhantes aos verificados em adultos. 
Estudo realizado por nosso grupo, com hipertensos adultos, demonstrou que um programa de exercícios domiciliar de curto prazo não supervisionado, composto por trabalho de flexibilidade e caminhada de 30 minutos e frequência de três vezes por semana, foi capaz de reduzir os níveis pressóricos e melhorar indicadores de aptidão física em apenas quatro meses. ${ }^{32}$ Os mesmos indivíduos foram acompanhados por 16 meses e o programa domiciliar mostrou-se novamente efetivo em reduzir a PA em longo prazo ou, pelo menos, manter os efeitos adquiridos no começo da rotina de caminhadas. ${ }^{33}$ Sugeriuse que, ao menos nos primeiros meses, as reduções pressóricas teriam sido mediadas por mecanismos relacionados ao exercício, já que alterações no peso e em variáveis antropométricas só ocorreram após 6 meses de intervenção. Esse fato corrobora a ideia de que programas de controle do peso que não incluem atividades físicas parecem não ter o mesmo efeito em variáveis hemodinâmicas. Watts e colaboradores, ${ }^{34}$ por exemplo, demonstraram que a intervenção nutricional por si só não seria capaz de gerar redução na PA e frequência cardíaca, o que seria possível apenas com programas combinados com exercícios físicos ou baseados exclusivamente em atividade física.

\section{Resistência à insulina e diabetes}

A principal função metabólica da insulina é a captação da glicose pelas células. Esse hormônio tem também ação hemodinâmica capaz de promover a vasodilatação e o aumento de fluxo sanguíneo, ao induzir a produção de óxido nítrico no endotélio, além disso favorece o recrutamento de capilares e o aumento do fluxo microvascular e da oferta não só da insulina, mas também do seu substrato, a glicose, para a musculatura esquelética e outros tecidos-alvo. ${ }^{35}$ A resistência à insulina e à disglicemia são dois dos diversos fatores que podem estar presentes na SM. Em indivíduos obesos com resistência insulínica, há um elevado nível de ácidos graxos livres com aumento da oxidação de lipídios e pela menor captação de glicose, uma redução da oxidação de glicose. De forma compensatória e secundária à menor captação de glicose, observa-se hiperinsulinemia. Precocemente, a resistência insulínica hepática promove nos períodos de jejum prolongado, como no noturno, maior gluconeogênese e elevações discretas da glicemia (glicemia de jejum alterada). A hiperinsulinemia e o aumento dos ácidos graxos livres podem por si só aumentar a atividade do sistema nervoso simpático e promover elevações da PA. À medida que a hiperinsulinemia não mantém mais os níveis de glicemia em valores normais, e o deficit de secreção relativa de insulina se instala, o diagnóstico de intolerância à glicose ou DM2 pode ser feito. ${ }^{20}$

Existem muitas evidências de que a atividade física melhora a sensibilidade do músculo esquelético à insulina. ${ }^{36}$ Sendo assim, diversas pesquisas têm encontrado uma associação negativa entre a prática regular de exercício físico e indicadores como glicemia e insulinemia de jejum. Alguns estudos indicam a permanência desses efeitos, mesmo quando os resultados são corrigidos para o peso corporal e o índice de massa corporal, reforçando a ideia de que o exercício físico atua sobre a resistência à insulina, independentemente da perda de peso. ${ }^{20}$

Tem sido demonstrado que o benefício da atividade física sobre a sensibilidade à insulina pode ser alcançado tanto por meio do exercício aeróbico como pela prática de exercícios resistidos. ${ }^{37}$ Porém, os mecanismos de ação parecem ser diferentes; enquanto os aeróbios atuam na redução ponderal, os exercícios de força são importantes para o controle glicêmico, principalmente em idosos que têm diminuição da força e massa muscular, afetando o metabolismo energético e quando realizado sistematicamente também pelo aumento da massa muscular. Sendo assim, a combinação dessas duas modalidades de atividade física é recomendada para prevenção e controle do diabetes tipo 2 .

Os efeitos de uma sessão de exercício 
aeróbio sobre a sensibilidade à insulina variam de acordo com a duração, intensidade e dieta posterior, podendo perdurar de 24 a 72 horas. Assim, as pesquisas indicam que os efeitos duradouros proporcionados pelos exercícios físicos na sensibilidade à insulina são dependentes da regularidade e manutenção da prática de atividade física. ${ }^{38}$ Por outro lado, a dieta hipocalórica, ao permitir redução ponderal, é capaz de aumentar a tolerância à glicose e a ação da insulina. Sendo assim, a associação entre exercício físico e dieta hipocalórica pode proporcionar ganhos maiores no tocante à sensibilidade à insulina, beneficiando o indivíduo com SM.

\section{Normas para prescrição do exercício físico no indivíduo com síndrome metabólica}

Um programa de exercícios direcionado ao indivíduo com SM deve conter, em geral, as características de um programa de exercícios aplicado a indivíduos saudáveis que desejam aprimorar a aptidão física. Nesse sentido, a intervenção deve ser composta por três componentes essenciais, a saber: treinamento aeróbio, treinamento de força e treinamento de flexibilidade.

A seguir, apresentaremos algumas normas de prescrição do exercício, tomando como base as diretrizes de prescrição do American College of Sports Medicine (ACSM) ${ }^{23}$ Embora o treinamento aeróbio e de força atuem de forma direta nos diferentes indicadores da síndrome metabólica, exercícios de flexibilidade também podem ser adicionados como parte integrante de um programa de condicionamento geral, visto os efeitos na melhora da amplitude de movimento e prevenção de dores lombares, entre outros aspectos.

No que diz respeito às normas de prescrição do exercício, diversos aspectos devem ser considerados. Geralmente o portador de SM não é bem-condicionado e apresenta excesso de peso corporal. Nesse caso, atividades como caminhada, ciclismo ao ar livre em cicloergômetro ou mesmo no meio aquático são aconselhados. Para esses indivíduos, o trabalho aeróbio deve ser iniciado em uma intensidade moderada, ou seja, $40 \%$ a $60 \%$ do consumo de oxigênio de reserva $\left(\mathrm{VO}_{2} \mathrm{R}\right)$ ou da frequência cardíaca de reserva (FCR). A duração pode ser iniciada com 20 minutos, evoluindo até 60 minutos, conforme a melhora da condição física do praticante. Para os mais condicionados, a corrida pode ser uma opção e o trabalho pode iniciar com intensidades entre 50\% e $75 \%$ do $\mathrm{VO}_{2} \mathrm{R}$ ou FCR, durante 30 a 60 minutos. É importante notar que, inicialmente, devese enfatizar a duração, para depois priorizar aumentos na intensidade do esforço. Em geral, essa conduta tende a tornar os programas de exercícios menos sacrificantes e aborrecidos, contribuindo com maior adesão dos praticantes. A frequência semanal deve ser de no mínimo três vezes por semana. Contudo, alterações mais significativas na aptidão tendem a ocorrer quando as sessões de exercício são realizadas de quatro a seis vezes por semana.

No caso do treinamento de força, o programa deveria incluir oito a dez exercícios para os principais grupamentos musculares requeridos nas atividades diárias. Para cada exercício, três séries de 10 a 12 repetições são habitualmente recomendadas. O praticante não necessita realizar trabalhos vigorosos para obter benefícios. Nesse caso, a carga inicial pode ser ajustada para realizar dez repetições. Quando o praticante conseguir realizar 12 repetições e sentir que a carga está leve pode aumentá-la, ou seja, não é necessário realizar repetições máximas. Aliás, a fadiga é um aspecto que deve ser evitado, por gerar desconforto desnecessário. Quanto ao intervalo entre séries, deve ser adequado para permitir uma correta recuperação do praticante - em geral, varia de 2 a 3 minutos. Contudo, esse tempo poderá ser modificado conforme a percepção de cansaço. Por fim, a frequência semanal deveria ser de pelo menos duas a três vezes, podendo chegar a cinco ou seis vezes, conforme a condição física e respostas individuais exibidas ao longo do 
treinamento.

Embora o treinamento de flexibilidade não exerça efeito direto nos indicadores da síndrome metabólica, deveria integrar um programa de exercícios direcionado à promoção da saúde. $\mathrm{O}$ trabalho de flexibilidade deveria envolver, preferencialmente, os músculos e articulações do ombro, tronco, quadril, joelhos e tornozelos. Não é necessário sentir dor durante a realização dos alongamentos, conduzidos até um ângulo em que se verifique leve desconforto. Com a evolução do condicionamento, os exercícios poderão ser sustentados com maior grau de desconforto, sem, contudo, chegar ao ponto de ocasionar dor. Deve-se repetir de três a cinco vezes cada alongamento, durante 15 a 30 segundos. Para iniciantes que possuem pouca flexibilidade, alongamentos com 10 segundos de duração podem ser aconselhados. Uma frequência semanal de três a cinco vezes é habitualmente recomendada.

\section{Considerações finais}

Para que os efeitos positivos do exercício sejam obtidos nos diferentes indicadores da $\mathrm{SM}$, o mesmo deve ser prescrito com bases metodológicas adequadas. Antes, porém, ao iniciar um programa de exercícios, o portador de SM deve ser submetido a uma avaliação clínica e preferencialmente a um teste cardiopulmonar de exercício. $\mathrm{Na}$ impossibilidade de se realizar este último procedimento, um teste de exercício máximo convencional com avaliação da resposta eletrocardiográfica ao esforço seria recomendado.

Como muitos indivíduos apresentam excesso de peso, cuidados preventivos para lesões osteoarticulares devem ser tomados. Nesse caso, atividades aeróbias como caminhar, pedalar, nadar, subir escadas e dançar devem ser recomendadas, adicionadas aos treinamentos de força e flexibilidade, sem necessidade do emprego de intensidades de trabalho elevadas. Por fim, a progressão das cargas de treinamento deve ser realizada de forma individualizada, obedecendo às respostas exibidas pelos praticantes. Nesse sentido, recomenda-se que o programa de exercícios seja elaborado por profissional com conhecimento nas áreas de fisiologia do exercício e treinamento físico, para que o praticante possa se beneficiar dos efeitos positivos do exercício com o menor risco possível.

\section{Referências}

1. Adair LS. Child and adolescent obesity: epidemiology and developmental perspectives. Physiol Behav. 2008;94(1):816. http://dx.doi.org/10.1016/j. physbeh.2007.11.016

2. Marquezine GF, Oliveira CM, Pereira AC, Krieger JE, Mill JG. Metabolic syndrome determinants in an urban population from Brazil: social class and gender-specific interaction. Int J Cardiol. 2008;129(2):259-65. http://dx.doi.org/10.1016/j.ijcard.2007.07.097

3. Brasil. Ministério da Saúde. VIGITEL Brasil 2010. Vigilância de fatores de risco e proteção para doenças crônicas por inquérito telefônico [acesso 10 dez 2013]. Disponível em: http:// bvsms.saude.gov.br/bvs/publicacoes/ vigitel_2010.pdf.

4. Alberti KG, Zimmet PZ. Definition, diagnosis and classification of diabetes mellitus and its complications. Part 1: diagnosis and classification of diabetes mellitus provisional report of a WHO consultation. Diabet Med. 1998;15(7):539-53. http://dx.doi.org/10.1002/ (SICI)1096-9136(199807)15:7<539::AIDDIA668>3.0.CO;2-S

5. Cleeman JI, Grundy SM, Becker D, Clark LT, Cooper RS, Denke MA, et al. Executive summary of the Third Report of the National Cholesterol Education Program (NCEP) expert panel on detection, evaluation, and treatment of high blood cholesterol in adults (Adult Treatment Panel III). Jama-J Am Med Assoc. 2001;285(19):2486-97.

6. Alberti KGMM, Zimmet P, Shaw J. The metabolic syndrome - a new worldwide definition. Lancet. 2005;366(9491):105962. http://dx.doi.org/10.1016/S01406736(05)67402-8

7. Alberti KG, Eckel RH, Grundy SM, Zimmet PZ, Cleeman JI, Donato KA, et al. Harmonizing the metabolic syndrome: a joint interim statement of the International Diabetes Federation Task Force on Epidemiology and Prevention; National Heart, Lung, and Blood Institute; American Heart Association; World Heart Federation; International Atherosclerosis Society; and 
International Association for the Study of Obesity. Circulation. 2009;120(16):1640-

5. http://dx.doi.org/10.1161/

CIRCULATIONAHA.109.192644

8. Cook DG, Mendall MA, Whincup PH, Carey IM, Ballam L, Morris JE, et al. C-reactive protein concentration in children: relationship to adiposity and other cardiovascular risk factors. Atherosclerosis. 2000;149(1):139-50.

9. Olson TP, Dengel DR, Leon AS, Schmitz $\mathrm{KH}$. Changes in inflammatory biomarkers following one-year of moderate resistance training in overweight women. Int J Obes (Lond). 2007;31(6):996-1003. http://dx.doi. org/10.1038/sj.ijo.0803534

10. Juhola J, Magnussen CG, Viikari JS, Kahonen M, Hutri-Kahonen N, Jula A, et al. Tracking of serum lipid levels, blood pressure, and body mass index from childhood to adulthood: The Cardiovascular Risk in Young Finns Study. J Pediatr. 2011;159(4):584-90. http://dx.doi.org/10.1016/j.jpeds.2011.03.021

11. Flynn MA, McNeil DA, Maloff B, Mutasingwa D, Wu M, Ford C, et al. Reducing obesity and related chronic disease risk in children and youth: a synthesis of evidence with 'best practice' recommendations. Obes Rev. 2006;7 Suppl 1:7-66. http://dx.doi.org/10.1111/j.1467789X.2006.00242.x

12. Lavelle HV, Mackay DF, Pell JP. Systematic review and meta-analysis of school-based interventions to reduce body mass index. J Public Health (Oxf.). 2012;34(3):360-9. http:// dx.doi.org/10.1093/pubmed/fdr116

13. Owens S, Gutin B, Allison J, Riggs S, Ferguson M, Litaker M, et al. Effect of physical training on total and visceral fat in obese children. Med Sci Sports Exerc. 1999;31(1):143-8.

14. Reilly JJ. Obesity in childhood and adolescence: evidence based clinical and public health perspectives. Postgrad Med J. 2006;82(969):429-37. http://dx.doi.org/10.1136/ pgmj.2005.043836

15. Després JP, Lemieux I. Abdominal obesity and metabolic syndrome. Nature. 2006;444(7121):881-7. http://dx.doi. org/10.1038/nature05488

16. Lemieux I, Pascot A, Prud'homme D, Almeras N, Bogaty P, Nadeau A, et al. Elevated C-reactive protein - Another component of the atherothrombotic profile of abdominal obesity. Arterioscler Thromb Vasc Biol. 2001;21(6):961-7.

17. Must A, Jacques PF, Dallal GE, Bajema CJ, Dietz WH. Long-term morbidity and mortality of overweight adolescents - a follow-up of the Harvard Growth Study of 1922 to 1935 . New Engl J Med. 1992;327(19):1350-5.

18. Wong PC, Chia MY, Tsou IY, Wansaicheong GK, Tan B, Wang JC, et al. Effects of a 12-week exercise training programme on aerobic fitness, body composition, blood lipids and C-reactive protein in adolescents with obesity. Ann Acad Med Singapore. 2008;37(4):286-93.

19. Leung FP, Yung LM, Laher I, Yao XQ, Chen ZY, Huang Y. Exercise, vascular wall and cardiovascular diseases: an update (Part 1). Sports Med. 2008;38(12):1009-24.

20. Trombeta IC, Batalha LC, Nunes CM, et al. Obesidade, Síndrome Cardiometabólica e Exercício Físico. In: Negrão CD, Barreto ACP. Cardiologia do exercício: do atleta ao cardiopata. Barueri: Manole; 2010. p. 400-28.

21. Fernandes RA, Christofaro DGD, Casonatto J, Codogno JS, Rodrigues E, Cardoso M, et al. Prevalência de dislipidemia em indivíduos fisicamente ativos durante a infância, adolescência e idade adulta. Arq Bras Cardiol. 2011;97(4):317-323. http://dx.doi.org/10.1590/ S0066-782X2011005000083

22. Sociedade Brasileira de Cardiologia. I Diretriz Brasileira de Diagnóstico e Tratamento da Síndrome Metabólica. Arq Bras Cardiol. 2005;84 (supl.1):1-28.

23. American College Of Sports Medicine. Diretrizes do ACSM para os Testes de Esforço e sua Prescrição. $8^{\mathrm{a}}$ ed. Rio de Janeiro: Guanabara Koogan, 2010. 272 p.

24. Sociedade Brasileira de Cardiologia. IV Diretriz Brasileira sobre dislipidemias e prevenção da aterosclerose departamento de aterosclerose. Arq Bras Cardiol. 2007;88 (supl.1):2-19.

25. Kelley GA, Kelley KS. Aerobic exercise and $\mathrm{HDL}_{2}-\mathrm{C}$ : A meta-analysis of randomized controlled trials. Atherosclerosis, 2006 Jan;184(1):207-15.

26. Grandjean PW, Crouse SF, Rohack JJ. Influence of cholesterol status on blood lipid and lipoprotein enzyme responses to aerobic exercise. J Appl Physiol. 2000 Aug;89(2):47280 .

27. Nunes APOB, Vinagre CG, Maranhão RC. Metabolismo de lípides plasmáticos e exercício físico. In: Negrão CD, Barreto ACP. Cardiologia do exercício: do atleta ao cardiopata. Barueri: Manole; 2010. p. 92-132.

28. Wang TJ, Vasan RS. Epidemiology of uncontrolled hypertension in the United States. Circulation. 2005;112(11):1651-62. 
29. Barroso WKS, Jardim PCBV, Vitorino PV, Bittencourt A, Miquetichuc F. Influência da atividade física programada na pressão arterial de idosos hipertensos sob tratamento não-farmacológico. Rev Assoc Med Bras 2008;54(4):328-33. http://dx.doi.org/10.1590/ S0104-42302008000400018

30. Whelton SP, Chin A, Xin X, He J. Effect of aerobic exercise on blood pressure: a metanalysis or randomized, controlled trials. Ann Intern Med.2002;136(7):493-503.

31. Rocchini AP, Katch V, Anderson J, Hinderliter J, Becque D, Martin M, et al. Blood pressure in obese adolescents: effect of weight loss. Pediatrics. 1988;82(1):16-23.

32. Farinatti PT, Oliveira RB, Pinto VL, Monteiro WD, Francischetti E. [Home exercise program: short term effects on physical aptitude and blood pressure in hypertensive individuals]. Arq Bras Cardiol. 2005; 84(6):473-9. http://dx.doi.org/10.1590/S0066782X2005000600008

33. Farinatti PTV, Oliveira RB, Luz LGO, Sanjuliani F, Monteiro WD. Effectiveness Of A Home-based Exercise Program On Fitness And Blood Pressure Of Hypertensive Patients. In: 59th Annual Meeting and 3rd World Congress on Exercise is Medicine of the American College of Sports Medicine,
2012, San Francisco. 59th Annual Meeting, 2012.

34. Watts K, Jones TW, Davis EA, Green D. Exercise training in obese children and adolescents: current concepts. Sports Med. 2005;35(5):375-92.

35. Rattigan S, Barrett EJ, Clark MG. Insulinmediated capillary recruitment in skeletal muscle: is this a mediator of insulin action on glucose metabolism? Curr Diab Rep. 2003;3(3):195-200. http://dx.doi.org/10.1007/ s11892-003-0063-4

36. Ivy JL. Muscle insulin resistance amended with exercise training: role of GLUT4 expression. Med Sci Sports Exerc. 2004;36(7):1207-11.

37. Evans EM, Racette SB, Peterson LR, Villaneal DT, Greiwe JS, Holloszy JO. Aerobic power and insulin action improve in response to endurance exercise training in healthy 77-87 yr olds. J Appl Physiol. 2005;98(1):40-5.

38. Colberg SR, Sigal RJ, Fernhall B, Regensteiner JG, Blissmer BJ, Rubin RR, et al. Exercise and type 2 diabetes: the American College of Sports Medicine and the American Diabetes Association: joint position statement. Diabetes Care. 2010;33(12):147-67. http:// dx.doi.org/10.2337/dc10-9990 


\section{Autores}

\section{Ada Fernanda P. S. Lima}

Laboratório de Atividade Física e Promoção da Saúde. Instituto de Educação Física e Desportos. Universidade do Estado do Rio de Janeiro. Rio de Janeiro, RJ, Brasil.

\section{Alexandre H. Okano}

Departamento de Educação Física. Centro de Ciências da Saúde. Universidade Federal do Rio Grande do Norte. Natal, RN, Brasil.

\section{Alice R. Sampaio}

Laboratório de Atividade Física e Promoção da Saúde. Instituto de Educação Física e Desportos. Universidade do Estado do Rio de Janeiro. Rio de Janeiro, RJ, Brasil.

\section{Ana Paula M. Guttierres}

Laboratório de Atividade Física e Promoção da Saúde. Instituto de Educação Física e Desportos. Universidade do Estado do Rio de Janeiro. Rio de Janeiro, RJ, Brasil.

\section{Astrogildo V. Oliveira Júnior}

Departamento de Educação Física e Folclore. Colégio Pedro II. Rio de Janeiro, RJ, Brasil.

\section{Brenno S. Silva}

Programa de Pós-graduação em Ciências da Atividade Física. Universidade Salgado de Oliveira. Niterói, RJ, Brasil.

\section{Daniel A. Bottino}

Laboratório de Pesquisas Clínicas e Experimentais em Biologia Vascular. Centro Biomédico.

Universidade do Estado do Rio de Janeiro. Rio de Janeiro, RJ, Brasil.

\section{Dionizio Mendes Ramos Filho}

Laboratório de Bioenergética e Fisiologia Mitocondrial. Centro de Ciências da Saúde. Universidade Federal do Rio de Janeiro. Rio de Janeiro, RJ, Brasil.

\section{Elirez B. Silva}

Laboratório de Pesquisa Clínica Escola. Departamento de Fisioterapia. Universidade Gama Filho. Rio de Janeiro, RJ, Brasil.

\section{Ercole C. Rubini}

Laboratório de Fisiologia do Exercício. Universidade Estácio de Sá. Rio de Janeiro, RJ, Brasil.

\section{Fabrício V. A. Vasconcellos}

Centro de Investigação, Formação, Inovação, Intervenção e Desporto. Faculdade de Desporto. Universidade do Porto. Porto, Portugal.

\section{Felipe A. da Cunha}

Programa de Pós-graduação em Ciências Médicas. Universidade do Estado do Rio de Janeiro. Rio de Janeiro, RJ, Brasil.

\section{Fernanda Monteiro}

Laboratório de Atividade Física e Promoção da Saúde. Instituto de Educação Física e Desportos. Universidade do Estado do Rio de Janeiro. Rio de Janeiro, RJ, Brasil.

\section{Flávia Porto}

Programa de Pós-graduação em Ciências do Exercício e do Esporte. Universidade Gama Filho. Rio de Janeiro, RJ, Brasil. 


\section{Gustavo C. Lopes}

Laboratório de Atividade Física e Promoção da Saúde. Instituto de Educação Física e Desportos. Universidade do Estado do Rio de Janeiro. Rio de Janeiro, RJ, Brasil.

\section{Jonas L. Gurgel}

Programa de Pós-graduação em Ciências

Cardiovasculares. Universidade Federal Fluminense.

Niterói, RJ, Brasil.

\section{Jonathan Myers}

VA Palo Alto Health Care System. Cardiology Division. Stanford University. Palo Alto, California, United States.

\section{Karynne Grutter}

Laboratório de Anatomia Humana. Universidade Castelo Branco. Rio de Janeiro, RJ, Brasil.

\section{Lenifran Matos-Santos}

Programa de Pós-graduação em Ciências da Atividade Física. Universidade Salgado de Oliveira. Niterói, RJ, Brasil.

\section{Lorena Paes}

Programa de Pós-graduação em Fisiopatologia Clínica e Experimental. Universidade do Estado do Rio de Janeiro. Rio de Janeiro, RJ, Brasil.

\section{Luciane P. da Costa}

Laboratório de Atividade Física e Promoção da Saúde. Instituto de Educação Física e Desportos. Universidade do Estado do Rio de Janeiro. Rio de Janeiro, RJ, Brasil.

\section{Luiz G. Kraemer-Aguiar}

Departamento de Medicina Interna. Faculdade de Ciências Médicas. Universidade do Estado do Rio de Janeiro. Rio de Janeiro, RJ, Brasil.

\section{Matheus R. Hausen}

Laboratório de Atividade Física e Promoção da Saúde. Instituto de Educação Física e Desportos. Universidade do Estado do Rio de Janeiro. Rio de Janeiro, RJ, Brasil.

\section{Paulo T. V. Farinatti}

Laboratório de Atividade Física e Promoção da Saúde. Instituto de Educação Física e Desportos. Universidade do Estado do Rio de Janeiro. Rio de Janeiro, RJ, Brasil.

\section{Rafael A. Montenegro}

Programa de Pós-graduação em Fisiopatologia Clínica e Experimental. Universidade do Estado do Rio de Janeiro. Rio de Janeiro, RJ, Brasil.

\section{Raul A. Freire}

Programa de Pós-graduação em Ciências da Atividade Física. Universidade Salgado de Oliveira. Niterói, RJ, Brasil.

\section{Renato O. Massaferri}

Programa de Pós-graduação em Ciências da Atividade Física. Universidade Salgado de Oliveira. Niterói, RJ, Brasil.

\section{Ricardo B. Oliveira}

Laboratório de Atividade Física e Promoção da Saúde. Instituto de Educação Física e Desportos. Universidade do Estado do Rio de Janeiro. Rio de Janeiro, RJ, Brasil.

\section{Ricardo G. Cordeiro}

Programa de Pós-graduação em Ciências da Atividade Física. Universidade Salgado de Oliveira. Niterói, RJ, Brasil.

\section{Sérgio Machado}

Programa de Pós-graduação em Ciências da Atividade Física. Universidade Salgado de Oliveira. Niterói, RJ, Brasil.

\section{Taciana Pinto}

Laboratório de Atividade Física e Promoção da Saúde. Instituto de Educação Física e Desportos. Universidade do Estado do Rio de Janeiro. Rio de Janeiro, RJ, Brasil.

\section{Tainah P. Lima Monteiro}

Programa de Pós-graduação em Ciências Médicas. Faculdade de Ciências Médicas. Universidade do Estado do Rio de Janeiro. Rio de Janeiro, RJ, Brasil.

\section{Tânia M. P. F. Paschoalino}

Hospital Universitário Antônio Pedro. Universidade Federal Fluminense. Niterói, RJ, Brasil.

\section{Walace D. Monteiro}

Laboratório de Atividade Física e Promoção da Saúde. Instituto de Educação Física e Desportos. Universidade do Estado do Rio de Janeiro. Rio de Janeiro, RJ, Brasil. 